\title{
Creating helping posters for electronic labs
}

\author{
Jiménez-Fernández, Carlos J.; Parra, Pilar; Baena, Carmen; Valencia, Manuel \\ Dpto. de Tecnología Electrónica, Universidad de Sevilla / Instituto de Microelectrónica de Sevilla \\ (Universidad de Sevilla / CSIC) \\ Sevilla, España \\ cjesus@us.es
}

\begin{abstract}
A Project is presented whose aim was to develop a set of posters that, as small tutorials, shows in a very visual way what are the basic task to do in a laboratory of electronic. These posters are directed to students of several subjects and degrees. Poster has been selected as didactic material because it is a low cost, friendly and easy method to recall information and to present the contents with explanations. Eight posters have been made with topics like the use of electronic instruments, circuit assembly, circuit verification, measurement and troubleshooting.
\end{abstract}

Keywords-Didactic material, Work on laboratory, Posters, Digital and analog electronic teaching.

\section{INTRODUCTION}

This work was developed as a Teaching Innovation Project in the University of Seville. The Project has been implemented in two Centers: the Escuela Politécnica Superior (EPS) and the Escuela Técnica Superior de Ingeniería Informática (ETSII). More precisely, it has been addressed to the following degrees: Grado en Ingeniería Electrónica Industrial (EPS) and Grado en Ingeniería Informática (ETSII). The Project has been developed by professors belonging to the Departamento de Tecnología Electrónica. The targets of the work are several subjects in the degrees which have some common features: 1/ the laboratory practices must be developed with basic electronic equipment (power supplies, oscilloscopes) by students who have no previous experience or very little, and 2/ the attendance of the students to the different laboratory sessions is not continuous in time, so, some of the skills they get in a specific session can be forgotten by many of them before they reach next one. This Project aims to find a solution to this problem.

At the beginning of the course, the students have been trained, in specific sessions to use the electronic instruments they are going to need afterwards, during the course.

The necessity of this Project came out from the observance of one important fact. Most of the students forget what they learned at the preparatory session or at least they need some help to remember it. This lack of knowledge could make them not to reach the proposed goals in the following laboratory sessions.
People who collaborated in the Project were a group of eight, all of them, working as teachers of the involved subjects. They selected eight fields of special interest. The method chosen for the transmission of information was a set of posters. The contents of every poster were selected by the team and the design/review/redesign tasks were collaborative. Although the final posters come from different authors the collaborative work allowed homogeneity in colors and graphic elements.

The set of posters have been thought to transmit and remind to the students basic information about the laboratory equipment, the circuits implementation and the necessary techniques to verify their operation as well as to measure different parameters.

As the laboratory sessions take place in four different laboratories in two different centers, four set of posters needed to be implemented, one for each laboratory.

This paper is organized as follows, in Section II the information included in posters is presented, in section III the contents of every poster is discussed and the posters themselves are shown, and in Section IV a summary with the main conclusions of the work is included.

\section{POSTER DESCRIPTIONS}

The basic information that has been considered essential and, hence, included in the posters can be classified in groups: three of the posters are directed to the use of the laboratory instruments, other two to the implementation of circuits, two more for the tasks of verification and measurement of parameters, and one to troubleshooting.

In every poster, data are presented in a similar style. Processes are shown decomposed in several steps in order to ease the remembrance and comprehension of the concepts, and also, to promote the students independence, making them able to solve, on their own, the gap between what they remember and what they should know. In a very concise way, the students receive the information in a short list of ordered instructions.

The same palette of colors has been chosen to mark each of the steps in the posters so that they have a homogeneous appearance when they are exposed in the laboratory walls. All the posters have been color printed and their light support make them cheaper and suitable for transport and placement. 
In the following paragraphs, the main details of every poster will be discussed.

\section{Power supply and multimeter}

This poster presents two instruments that are basic in the electronic laboratory: the multimeter and the power supply. The first one is used to measure some electric magnitudes like voltage, current, resistance, etc. The second one is used as power source and can generate constant voltages. Figure 1 shows the poster.

In relation to the multimeter, the poster shows an existing model included in the laboratories where the students do their practices. The image is labeled to highlight the different connectors. The measurement methodology is explained through a series of required steps.

In relation to the power supply, the same structure has been used: an image of the instrument and the necessary steps to use it. Two typical configurations are also presented: digital circuits and operational amplifiers.

\section{Function generator}

This poster shows the function generator. This device, in some of the cases, requires that the user work with the oscilloscope to set properly the parameters of the generated signals. Therefore, it is important to describe to the students the adequate configuration of the generator as well as the sequence they have to follow and the necessity of using additional equipment. Poster is shown in Figure 2.
As it can be seen, in the upper part of the poster the three possibilities of waveform have been enumerated, while configurable parameters (amplitude, frequency and dc offset) are presented over the drawing of a periodic signal. Under this information a sequence of steps is shown in the poster, although this sequence is not necessarily fixed, it has been recommended to configure in first place all those parameters that can be set without the use of the oscilloscope. More precisely, the established sequence is: 1) configuration of the type of signal, 2) configuration of the frequency, 3) connection the generator to the oscilloscope, and 4) setting the amplitude and the dc offset (using the oscilloscope).

In the poster, an image with a generator is used to present where the parameters aforementioned can be modified.

\section{Oscilloscope}

The third poster is about the oscilloscope. With an oscilloscope, signals can be observed in real time on a screen and voltages and time parameters can be measured with precision. The number and complexity of the tasks that can be performed with an oscilloscope is high. A good configuration of the oscilloscope is essential to get a good representation of the signals. This is why a specific poster has been dedicated to it. In Figure 3 the poster is shown.

A labeled image of an oscilloscope is presented in the upper side of the poster. Next to it, another image shows what are the connectors and the way they must be used in order to display the signal.

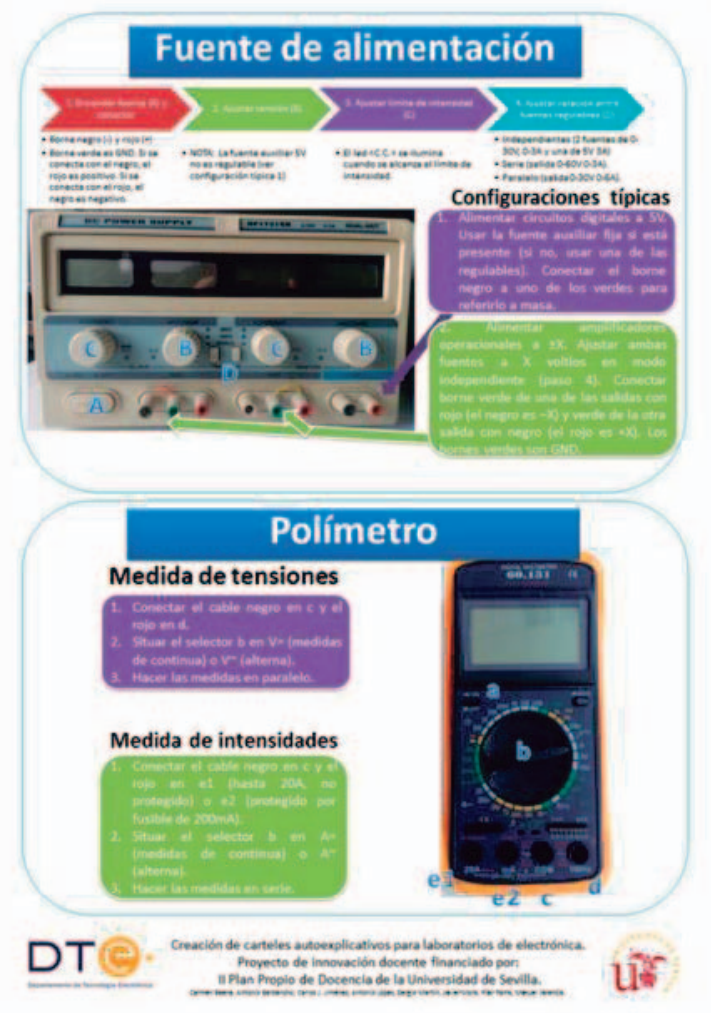

Figure 1: Poster 1: Multimeter and DC Power Supply

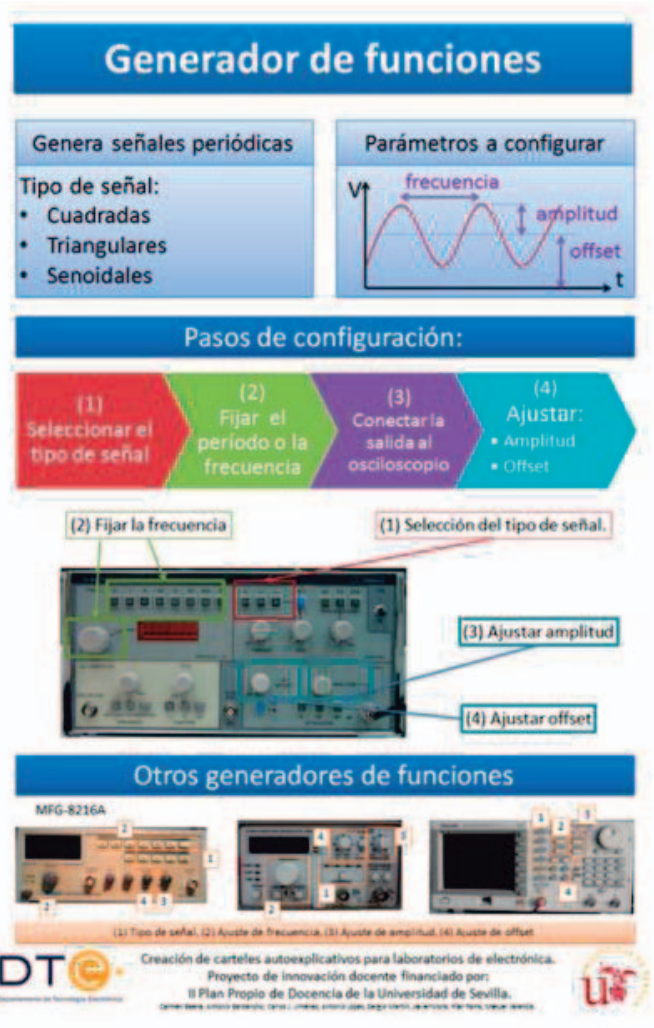

Figure 2: Poster 2: Function Generator 


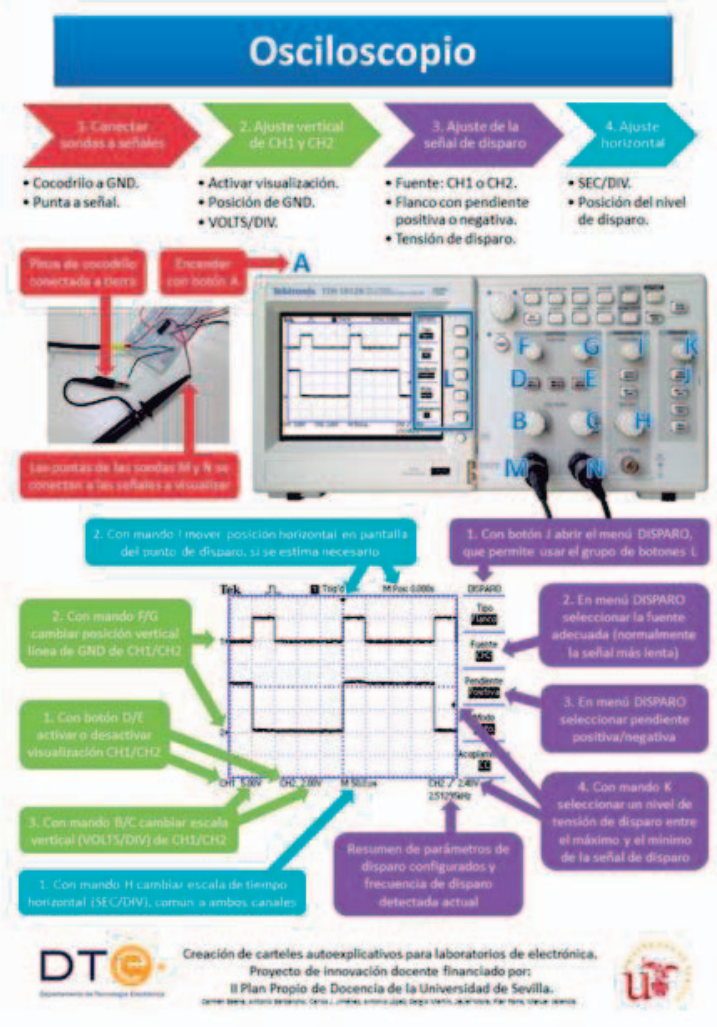

Figure 3: Poster 3: Oscilloscope

Most important steps are also presented. 1) Connection between oscilloscope and signals; 2) Vertical adjustment of each channel (voltage scale and ground line); 3) Trigger adjustment (selection of: trigger signal, type of trigger and trigger level); and 4) Horizontal adjustment (time scale and horizontal trigger).

\section{Digital circuit assembly}

The target of this poster (Figure 4) is detailing the procedure of digital circuit assembly.

\section{A four-step procedure is presented.}

First, the circuit board and its internal connections are presented. On a picture, the position and orientation recommended for circuits is shown and the proper way of connecting the power supply is explained too.

The second step helps to identify the inputs, the outputs and the internal nodes with the pins of the integrated circuit. It is recommended to write down the pin number on a circuit scheme as shown.

The third step presents how to connect the pins one each other using wires according to the previous scheme.

Finally, it is detailed how to work in order to start the operation of the circuit, how to apply the stimuli in the inputs using the power supply or the function generator. Finally, it is emphasized that the use of the oscilloscope will be necessary to verify the circuit operation.

\section{Montaje de circuitos digitales}

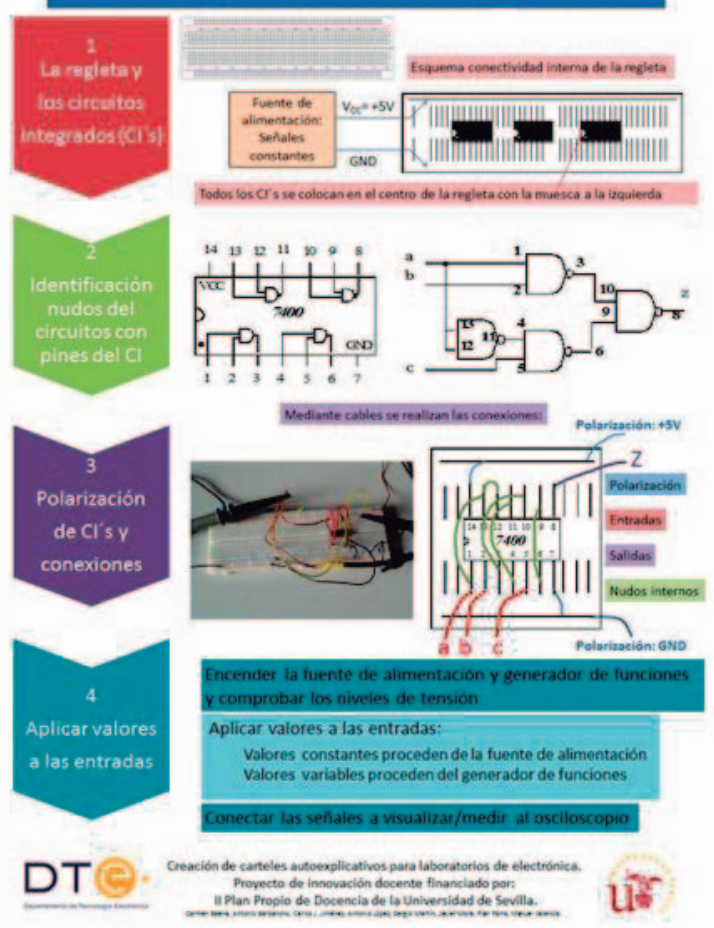

Figure 4: Poster 4: Digital circuit assembly

\section{Analog circuit assembly}

In this poster, it is shown the relation between the schematic of an analog circuit and its implementation using integrated circuits, as well as the connections needed with the measuring instrumental. In the figure 5 the poster can be seen.

The starting point is the schematic of a specific circuit. This one must be simple enough in order to pupils do not be lost in the structure of it but, simultaneously, the main details in an experimental assembly must be taken into account. These are: the physical assembly of the circuit using integrated circuits and connections, the power supply, the excitation in the inputs of the circuit and the measurement of the interesting signals.

In the physical assembly of the circuit, it is needed to dispose the different components on the circuit board with as minimum number of electrical connections as possible and they must interfere as less as possible in the installation itself (short and straight connections avoiding crossing and overlapping above the components). To show this idea, an enlarged photograph of a circuit assembly on a circuit board is included.

For the power supply, it is needed to connect the power source with the right nodes of the circuit. For this, the positions of the connectors in the power source and the nodes in the circuit where they must be connected are identified. This is shown by a photo of the connectors of the power source and the labels of the connection nodes on the image of the circuit, in the example: $+15 \mathrm{~V},-15 \mathrm{~V}$ y GND. 


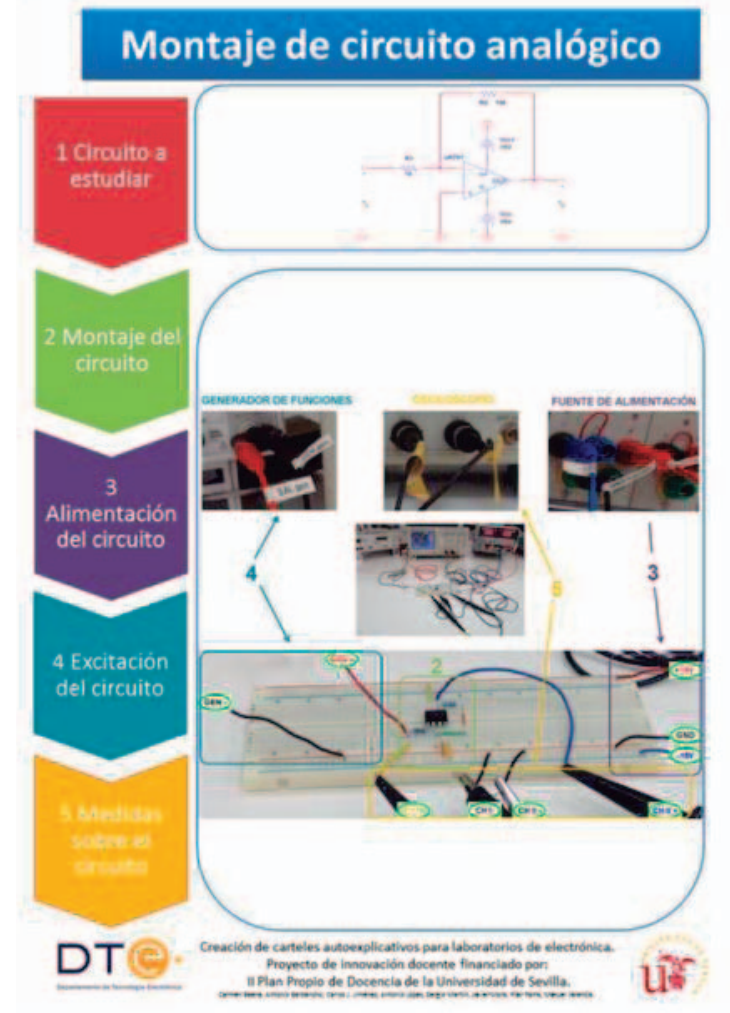

Figure 5: Poster 5: Analog circuit assembly

To excite the circuit, it is needed to connect the function generator to the adequate nodes. For this, the positions of the connectors in the already said instrument and the nodes in the circuit where they must be connected are identified. Again, a photo is used to show the connectors of the function generator and the labels of the connection nodes on the image of the circuit, in the example: GEN+y GEN-.

Similarly, it has been proceed with the oscilloscope to show how to measure the interesting signals.

\section{Test and verification tasks}

The final target of the majority of the experimental jobs is the check the proper operation of the circuit under study. The goal of the poster "Test procedure" is to give a simple idea about how to develop this task in a tidy way in the case of digital circuits.

The most suitable instrument to verify digital circuits is the logic analyzer. Anyway, it is not common to dispose it in the laboratories where these posters will be exposed, but they have typical electronic instruments: power supply, function generator and oscilloscope. They also have LEDs as simple elements to visualize 0 's and 1's. That is the reason why the poster is focused on the process of test using only those instruments. In the figure 6 the poster is shown.

The main guideline which is expressed in the poster is the idea of organizing the steps in the test, but besides, some peculiarities are taken into account like: the case of a

\section{Proceso de test (caso digital)}

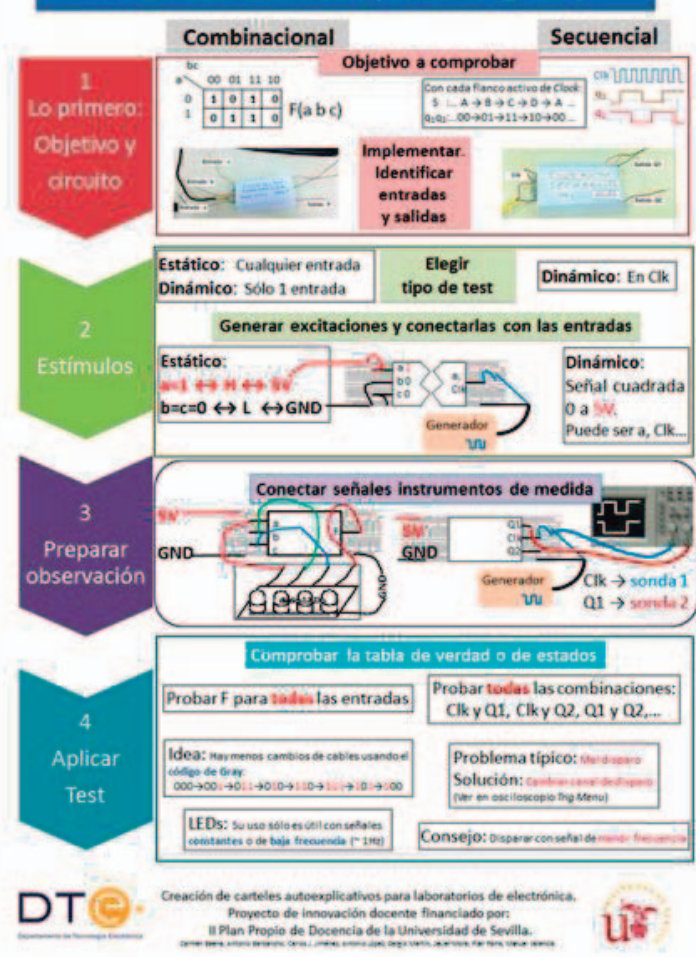

Figure 6: Poster 6: Test procedure (digital)

combinational circuit opposite to sequential one, use of static stimuli opposite to dynamic ones, and the use of the oscilloscope opposite to the LEDs. The steps in the test are:

1) The previous work is centered in two aspects: knowing the function to be verified and its implementation, object to be tested. In relation to the first one, it is about the verification of the theoretical functionality described by the Karnaugh map or transition table of states/outputs, depending on the case of the function. Regarding to the implementation, the most significant in the test is the relation input/output of the circuit and not that much the details inside the circuit, so in the implementation it must be clearly defined what signals are the inputs and what the outputs.

2) To get ready the stimuli to the circuit, both the static and the dynamic ones. In general, static stimuli can be disposedthrough the power lines GND and $+5 \mathrm{~V}$ - or dynamic stimuli, in this case, with the limitation of having only one square signal between 0 and $+5 \mathrm{~V}$. This signal will be used as clock in the sequential circuits, having static values for the rest of the inputs. In the combinational circuits, the dynamic signal can be used for one of the inputs while the rest will be excited with static values, or static values for all the inputs can be another option.

3) To prepare the analysis of the signals. The signals to be analyzed will be connected to the measurement instruments. The type of stimuli, static or dynamic, decide the instrument to be used to observe the signals and how many signals can be 
analyzed simultaneously. In the static case, the use of LEDs allows for connecting many -even all- input/output signals of the combinational function. On the contrary, if one dynamic signal is used, it will be connected (this input) to the channel 1 of the oscilloscope and one of the outputs to the channel 2 and, in this way, they can be visualized simultaneously on the screen of the oscilloscope.

4) Finally, to apply the test and check the functions "objective" of the first point. In the case of combinational functions, the idea is to complete an exhaustive analysis testing all combinations of inputs, and, in the sequential one, the task is to check the clock, the state variables and the outputs in pairs. It is emphasized several features, like: to give the stimuli to the circuit using Gray code because the number of wire connection changes is decreased; the use of LEDs is restricted to the case of static stimuli or at rate close to $1 \mathrm{~Hz}$; in the case of sequential circuits, it is common to have problem with the triggering so, it is suggested to use the slowest signal to trigger the oscilloscope.

\section{Time measurements}

The time response of a digital circuit is related to the efficiency because it affects to the maximum operational rate. Therefore, it is essential to transmit to the students which is the correct procedure to measure. In the figure 7 , this poster is shown.

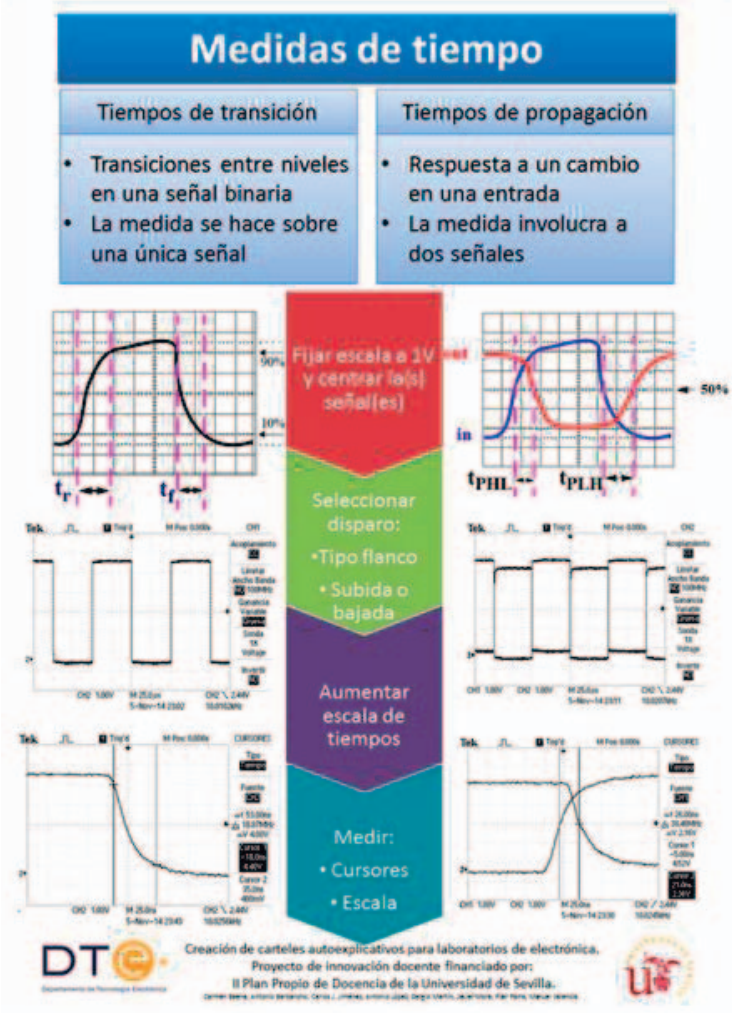

Figure 7: Poster 7: Time measurements
First, it is established the two types of time measurements: transition time and propagation time. So, the first part in the poster is dedicated to show the particular characteristics between them through two frames in parallel. This type of parallel presentation is kept along the poster in order to allow a better comparison between these very similar procedures but with little differences.

After the two first frames, two pictures are shown where it is marked the measurement to do. In the central part of the poster, it is presented a guide with the steps to follow. Simultaneously, on both sides of the poster they are shown the typical screens that should be obtained on the oscilloscope while the recommended steps are done. These are: 1) right selection of the scales and the position of the ground line in order to see the signals in the middle of the screen, and enlarge the precision of the measurement; 2) selection of the type of triggering which has to be by edge, rising or falling depending on the measurement to carry out; 3 ) Increase the time scale to get a correct measurement and 4) to measure.

\section{Troubleshooting: steps to resolve the mistakes}

One of the most difficult ideas to transmit to the students is the methodology to discover where the fault is when a circuit does not run properly. On this poster it has been exposed the procedure of looking for the possible elements which are failing. In the figure 8 , the poster is shown.

Five steps have been proposed that are exposed briefly

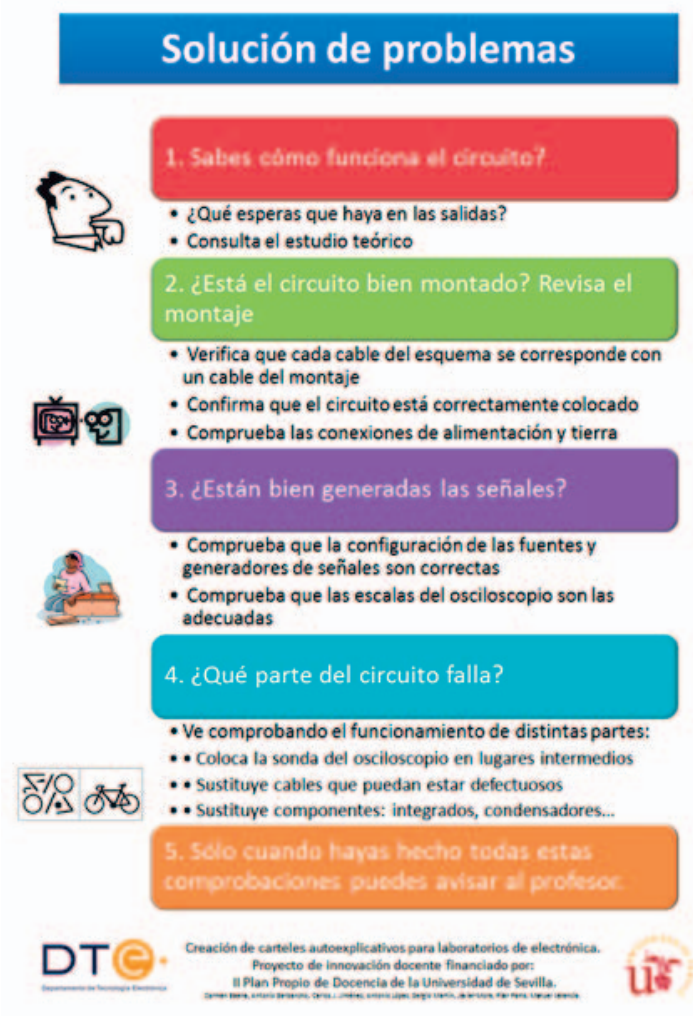

Figure 8: Poster 8: Troubleshooting 
next:

1) Knowledge of the proper functioning of the circuit. In many cases, the student does not know well the outputs that must be provided by the circuit he is verifying. Therefore, the first recommendation is that he has to know the expected outputs and he can make the comparison with the ones he is obtaining. To do that, he must take into account the document of the laboratory work and the theoretical study developed.

2) Check-up the circuit assembly. Once you are sure that the outputs do not match with the expected values, it is necessary to check the circuit assembly. This revision has different steps: check the connections among components, detection of inputs and output without connection, etc. In this point, it is recommended to use the scheme of the circuit assembly to be performed and compare it with the executed assembly.

3) Signal generation. This step is thought to check if the generated signals for the inputs are correct: the supply voltage and input voltages (both dc and ac). The oscilloscope must be used to verify the values of the signals.

4) Localization of the fault. In this step, a tracing of the signal from the input to the output is developed. To do that, the oscilloscope probe is connected to different nodes in the circuit. Based on the values on the intermediate nodes the place of the fault is discovered, as well as the possible causes (wrong connection, faulty components, etc.).

5) Call the teacher. This is the last step to take. For many students, this is considered as the first one, but it must be the last. The student must be able not only to set up the circuit assembly but also to verify the function in case of fault. So it is important to get into the habit of going to the teacher when there is no way to solve the problem by himself.

\section{RESULTS}

In this section, the final result of the project developed is presented. First, despite the difficulties due to the authors of the project are 8 people of 4 different subjects and they work in 2 different centers far away one from the other, a collaborative work has been done and concluded successfully.

Secondly, since the budget for the project was low, the posters have been impressed in A2 size color and using a light support in order to get an easy installation on the walls of the laboratories and to reduce the costs. Despite the economic difficulties, it was possible to complete 4 sets of the 8 posters that were installed in the 4 laboratories of the 2 centers. In the figures $8,9,10$ and 11 the photos of the 4 laboratories are shown.

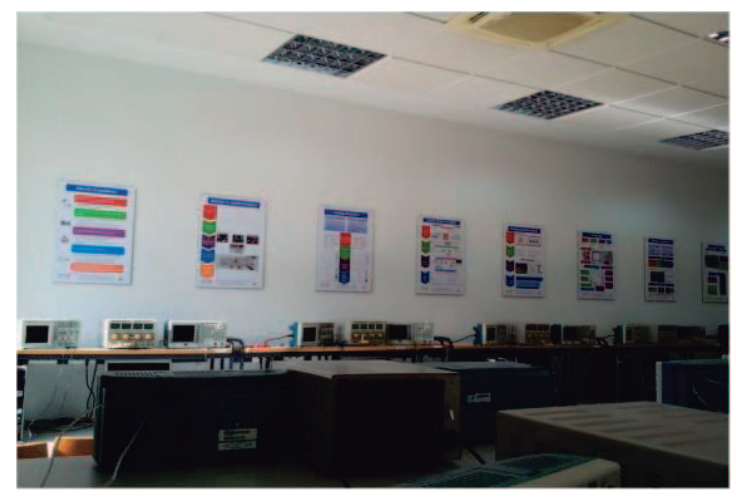

Figure 9: Posters in the ETSII (Lab 1)

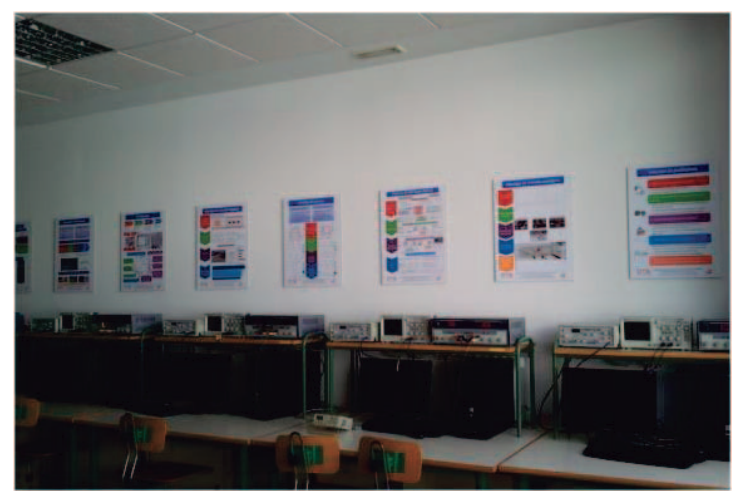

Figure 10: Posters in the ETSII (Lab 2)

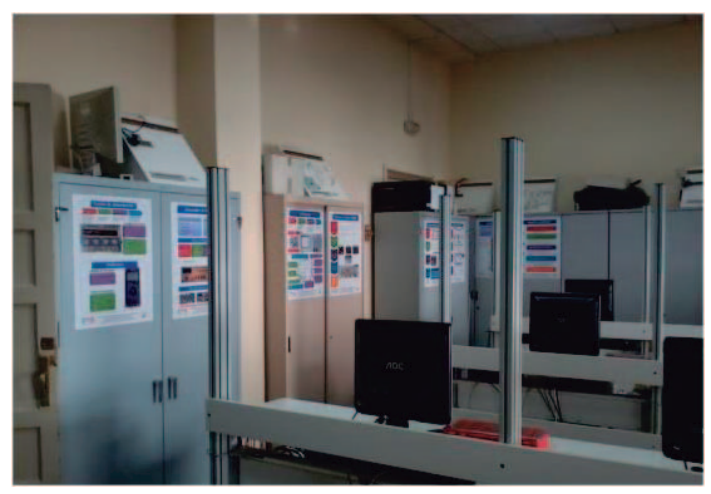

Figure 11: Posters in the EPS (Lab 1)

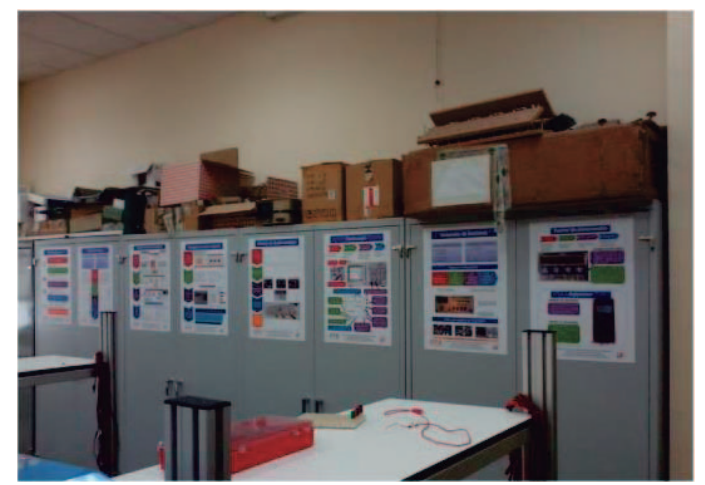

Figure 12: Posters in the ETSII (Lab 2) 


\section{CONCLUSIONS}

The result of the experience has been highly positive both the success in the development of the collaborative work, and the great reception among the students and the teachers has had the posters exposed in the laboratories. The didactic target of this work has been totally achieved because the posters have given an efficient way to remember the main procedures of the work in a laboratory. The student, at a glance, is able to remember the important details in the work he has to do without bothering the rest of the collages. Thus, the teacher is released from this task and he can invest the class time to present new concepts in each practice.

\section{ACKNOWLEDGEMENTS}

This Innovation Project has been developed thanks to the job of 8 teachers of the Tecnología Electrónica Department of the University of Seville. On the one hand, the authors of this article and, on the other hand, are: Sergio Martín Guillén, Antonio Barbancho Concejero, Antonio López Ojeda y Javier Mora Merchán.

The project, "Creating helping poster for electronic labs", in B mode innovation aid and teaching improvement, (call 2013-2014, Plan Propio), has been funded by the University of Seville. 